PROCEEDINGS OF THE

AMERICAN MATHEMATICAL SOCIETY

Volume 127, Number 5, Pages 1313-1322

S 0002-9939(99)04714-0

Article electronically published on January 27, 1999

\title{
DEGENERATIONS FOR MODULES OVER REPRESENTATION-FINITE ALGEBRAS
}

\author{
GRZEGORZ ZWARA
}

(Communicated by Ken Goodearl)

\begin{abstract}
Let $A$ be a representation-finite algebra. We show that a finite dimensional $A$-module $M$ degenerates to another $A$-module $N$ if and only if the inequalities $\operatorname{dim}_{K} \operatorname{Hom}_{A}(M, X) \leq \operatorname{dim}_{K} \operatorname{Hom}_{A}(N, X)$ hold for all $A$-modules $X$. We prove also that if $\operatorname{Ext}_{A}^{1}(X, X)=0$ for any indecomposable $A$-module $X$, then any degeneration of $A$-modules is given by a chain of short exact sequences.
\end{abstract}

\section{INTRODUCTION AND MAIN RESULTS}

Let $A$ be a finite dimensional associative $K$-algebra with an identity over an algebraically closed field $K$. If $a_{1}=1, \ldots, a_{n}$ is a basis of $A$ over $K$, we have the structure constants $a_{i j k}$ defined by $a_{i} a_{j}=\sum a_{i j k} a_{k}$. The affine variety $\bmod _{A}(d)$ of $d$-dimensional unital left $A$-modules consists of $n$-tuples $m=\left(m_{1}, \ldots, m_{n}\right)$ of $d \times d$ matrices with coefficients in $K$ such that $m_{1}$ is the identity matrix and $m_{i} m_{j}=$ $\sum a_{i j k} m_{k}$ holds for all indices $i$ and $j$. The general linear group $\mathrm{Gl}_{d}(K)$ acts on $\bmod _{A}(d)$ by conjugation, and the orbits correspond to the isomorphism classes of $d$-dimensional modules (see [11]). We shall agree to identify a $d$-dimensional $A$ module $M$ with the point of $\bmod _{A}(d)$ corresponding to it. We denote by $\mathcal{O}(M)$ the $\mathrm{Gl}_{d}(K)$-orbit of a module $M$ in $\bmod _{A}(d)$. Then one says that a module $N$ in $\bmod _{A}(d)$ is a degeneration of a $\operatorname{module} M$ in $\bmod _{A}(d)$ if $N$ belongs to the Zariski closure $\overline{\mathcal{O}(M)}$ of $\mathcal{O}(M)$ in $\bmod _{A}(d)$, and we denote this fact by $M \leq_{\operatorname{deg}} N$. Thus $\leq_{\text {deg }}$ is a partial order on the set of isomorphism classes of $A$-modules of a given dimension. It is not clear how to characterize $\leq_{\text {deg }}$ in terms of representation theory.

There has been a work by S. Abeasis and A. del Fra [1], K. Bongartz [6], [9], [8], Ch. Riedtmann [13], and A. Skowroński and the author [15], [16], [17] and [18] connecting $\leq_{\text {deg }}$ with other partial orders $\leq_{\text {ext }}$ and $\leq$ on the isomorphism classes in $\bmod _{A}(d)$. They are defined in terms of representation theory as follows:

- $M \leq_{\text {ext }} N$ : $\Leftrightarrow$ there are modules $M_{i}, U_{i}, V_{i}$ and short exact sequences $0 \rightarrow$ $U_{i} \rightarrow M_{i} \rightarrow V_{i} \rightarrow 0$ in $\bmod A$ such that $M=M_{1}, M_{i+1}=U_{i} \oplus V_{i}, 1 \leq i \leq s$, and $N=M_{s+1}$ for some natural number $s$.

- $M \leq N: \Leftrightarrow[M, X] \leq[N, X]$ holds for all modules $X$.

Received by the editors May 6, 1997 and, in revised form, August 28, 1997.

1991 Mathematics Subject Classification. Primary 14L30, 16G60, 16G70.

(C)1999 American Mathematical Society 
Here and later on we abbreviate $\operatorname{dim}_{K} \operatorname{Hom}_{A}(X, Y)$ by $[X, Y]$. Then for modules $M$ and $N$ in $\bmod _{A}(d)$, the following implications hold:

$$
M \leq_{\text {ext }} N \Longrightarrow M \leq_{\text {deg }} N \Longrightarrow M \leq N
$$

(see [9], [13]). Unfortunately, the reverse implications are not true in general, and it would be interesting to find out when they are. K. Bongartz proved in [9] that it is the case for all representations of Dynkin quivers and the double arrow. Moreover, in [8] K. Bongartz proved that $\leq_{\operatorname{deg}}$ and $\leq$ coincide for all modules over tame concealed algebras. Recently, the author proved in [17] that $\leq$ and $\leq$ ext are also equivalent for all modules over representation-finite blocks of group algebras, and in [18] that $\leq_{\text {ext }}$ and $\leq_{\text {deg }}$ coincide for all modules over tame concealed algebras. The main aim of this paper is to prove the following theorem.

Theorem 1. Let $A$ be a representation-finite algebra and $M, N$ two modules with $M \leq N$. Then there are $A$-modules $Z, Z^{\prime}$, and two exact sequences

$$
0 \rightarrow N \rightarrow M \oplus Z \rightarrow Z \rightarrow 0 \quad \text { and } \quad 0 \rightarrow Z^{\prime} \rightarrow M \oplus Z^{\prime} \rightarrow N \rightarrow 0 .
$$

In [13] Riedtmann proved that each of the exact sequences $0 \rightarrow N \rightarrow M \oplus Z \rightarrow$ $Z \rightarrow 0$ and $0 \rightarrow Z^{\prime} \rightarrow M \oplus Z^{\prime} \rightarrow N \rightarrow 0$ implies that $M \leq_{\operatorname{deg}} N$. Hence we get the following fact which solves a long standing problem (see [13]).

Corollary. The partial orders $\leq$ and $\leq_{\operatorname{deg}}$ coincide for all modules over representation-finite algebras.

We note that for a representation-finite algebra $A$ we may deduce the dimension of the spaces $\operatorname{Hom}_{A}(M, N)$ from the Auslander-Reiten quiver of $A$ (see [10]), and hence it is rather easy to decide when $M \leq N$ for any $A$-modules $M$ and $N$.

There are many examples of representation-finite algebras for which the orders $\leq_{\text {deg }}$ and $\leq_{\text {ext }}$ are not equivalent (see [17]). Our second aim in this paper is to prove the following theorem.

Theorem 2. Let $B$ be an algebra and assume that $\operatorname{Ext}_{B}^{1}(X, X)=0$ for any indecomposable $B$-module $X$. Then the partial orders $\leq, \leq_{\operatorname{deg}}$ and $\leq_{\text {ext }}$ coincide for all $B$-modules.

It is well-known that every representation-directed algebra [14] satisfies the above condition. Hence, Theorem 2 extends the corresponding result by Bongartz proved in [9].

The paper is organized as follows. In Section 2 we fix the notation, recall the relevant definitions and facts, and prove some preliminary results on modules which we apply in our investigations. Section 3 is devoted to the proofs of Theorems 1 and 2 .

For basic background on the topics considered here we refer to [5], [9], [8], [11] and [14]. The results presented in this paper form a part of the author's doctoral dissertation written under the supervision of Professor A. Skowroński. The author gratefully acknowledges support from the Polish Scientific Grant KBN No. 2 PO3A 02008 .

\section{Preliminary Results}

2.1. Throughout the paper $A$ denotes a fixed finite dimensional associative $K$ algebra with an identity over an algebraically closed field $K$. We denote by $\bmod A$ the category of finite dimensional left $A$-modules and by $\operatorname{rad}(\bmod A)$ the Jacobson 
radical of $\bmod A$. By an $A$-module we mean an object from $\bmod A$. Further, we denote by $\Gamma_{A}$ the Auslander-Reiten quiver of $A$, and by $\tau=\tau_{A}$ and $\tau^{-}=\tau_{A}^{-}$ the Auslander-Reiten translations $D \operatorname{Tr}$ and $\operatorname{Tr} D$, respectively. We shall agree to identify the vertices of $\Gamma_{A}$ with the corresponding indecomposable modules. For a module $M$ we denote by $[M]$ the image of $M$ in the Grothendieck group $K_{0}(A)$ of $A$. Thus $[M]=[N]$ if and only if $M$ and $N$ have the same simple composition factors including the multiplicities.

2.2. Following [13], for $M, N$ from $\bmod A$, we set $M \leq N$ if and only if $[M, X] \leq$ $[N, X]$ for all $A$-modules $X$. The fact that $\leq$ is a partial order on the isomorphism classes of $A$-modules follows from a result by M. Auslander [3] (see also [6]). Observe that, if $M$ and $N$ have the same dimension and $M \leq N$, then $[M]=[N]$. Moreover, M. Auslander and I. Reiten have shown in [4] that, if $M$ and $N$ are $A$-modules with $[M]=[N]$, then for all nonprojective indecomposable $A$-modules $X$ and all noninjective indecomposable modules $Y$ the following formulas hold (see [12]):

$$
\begin{aligned}
{[X, M]-[M, \tau X] } & =[X, N]-[N, \tau X], \\
{[M, Y]-\left[\tau^{-} Y, M\right] } & =[N, Y]-\left[\tau^{-} Y, N\right] .
\end{aligned}
$$

Hence, if $[M]=[N]$, then $M \leq N$ if and only if $[X, M] \leq[X, N]$ for all $A$-modules $X$.

2.3. Let $M$ and $N$ be $A$-modules with $[M]=[N]$ and

$$
\Sigma: 0 \rightarrow D \rightarrow E \rightarrow F \rightarrow 0
$$

an exact sequence in $\bmod A$. Following [13] we define the additive functions $\delta_{M, N}$, $\delta_{M, N}^{\prime}$ and $\delta_{\Sigma}$ on $A$-modules $X$ as follows:

$$
\begin{aligned}
\delta_{M, N}(X) & =[N, X]-[M, X], \\
\delta_{M, N}^{\prime}(X) & =[X, N]-[X, M], \\
\delta_{\Sigma}(X) & =\delta_{E, D \oplus F}(X)=[D \oplus F, X]-[E, X], \\
\delta_{\Sigma}^{\prime}(X) & =\delta_{E, D \oplus F}^{\prime}(X)=[X, D \oplus F]-[X, E] .
\end{aligned}
$$

From the Auslander-Reiten formulas (2.2) we get the following very useful equalities:

$$
\delta_{M, N}(X)=\delta_{M, N}^{\prime}\left(\tau^{-} X\right), \quad \delta_{M, N}(\tau X)=\delta_{M, N}^{\prime}(X)
$$

for all $A$-modules $X$. Observe also that $\delta_{M, N}(I)=0$ for any injective $A$-module $I$, and $\delta_{M, N}^{\prime}(P)=0$ for any projective $A$-module $P$. In particular, the following conditions are equivalent:

(1) $M \leq N$,

(2) $\delta_{M, N}(X) \geq 0$ for all $X \in \Gamma_{A}$,

(3) $\delta_{M, N}^{\prime}(X) \geq 0$ for all $X \in \Gamma_{A}$.

2.4. For an $A$-module $M$ and an indecomposable $A$-module $Z$, we denote by $\mu(M, Z)$ the multiplicity of $Z$ as a direct summand of $M$. For a noninjective indecomposable $A$-module $U$, we denote by $\Sigma(U)$ an Auslander-Reiten sequence

$$
\Sigma(U): 0 \rightarrow U \rightarrow E(U) \rightarrow \tau^{-} U \rightarrow 0 .
$$


We shall need the following lemma.

Lemma 2.5. Let $M, N$ be A-modules with $[M]=[N]$ and $U$ an indecomposable A-module. Then:

(i) If $U$ is noninjective, then $\delta_{\Sigma(U)}(M)=\mu(M, U)$.

(ii) If $M \leq N$, then $\mu(N, U)-\mu(M, U) \leq \delta_{M, N}(U)+\delta_{M, N}^{\prime}(U)$.

Proof. If $U$ is noninjective, then the Auslander-Reiten sequence $\Sigma(U)$ induces an exact sequence

$$
0 \rightarrow \operatorname{Hom}_{A}\left(\tau^{-} U, M\right) \rightarrow \operatorname{Hom}_{A}(E(U), M) \rightarrow \operatorname{rad}(U, M) \rightarrow 0,
$$

and hence we get

$$
\left[U \oplus \tau^{-} U, M\right]-[E(U), M]=[U, M]-\operatorname{dim}_{K} \operatorname{rad}(U, M)=\mu(M, U) .
$$

This implies (i). Similarly, we have

$$
\left[U \oplus \tau^{-} U, N\right]-[E(U), N]=\mu(N, U) .
$$

Then we obtain

$$
\begin{aligned}
\mu(N, U)-\mu(M, U) & =\left(\left[U \oplus \tau^{-} U, N\right]-\left[U \oplus \tau^{-} U, M\right]\right)-([E(U), N]-[E(U), M]) \\
& =\delta_{M, N}^{\prime}(U)+\delta_{M, N}^{\prime}\left(\tau^{-} U\right)-\delta_{M, N}^{\prime}(E(U)) \\
& \leq \delta_{M, N}^{\prime}(U)+\delta_{M, N}^{\prime}\left(\tau^{-} U\right)=\delta_{M, N}^{\prime}(U)+\delta_{M, N}(U) .
\end{aligned}
$$

Assume now that $U$ is injective. Then $\operatorname{Hom}_{A}(U / \operatorname{soc}(U), M) \simeq \operatorname{rad}(U, M)$, and so

$$
[U, M]-[U / \operatorname{soc}(U), M]=\mu(M, U) .
$$

Similarly, we have

$$
[U, N]-[U / \operatorname{soc}(U), N]=\mu(N, U) .
$$

Therefore, we get

$$
\begin{aligned}
\mu(N, U)-\mu(M, U) & =([U, N]-[U, M])-([U / \operatorname{soc}(U), N]-[U / \operatorname{soc}(U), M]) \\
& =\delta_{M, N}^{\prime}(U)-\delta_{M, N}^{\prime}(U / \operatorname{soc}(U)) \leq \delta_{M, N}^{\prime}(U) \\
& =\delta_{M, N}^{\prime}(U)+\delta_{M, N}(U) .
\end{aligned}
$$

Hence, (ii) also holds.

We shall need also the following Lemma $(3+3+2)$ from $[2,(2.1)]$ and its direct consequence.

Lemma 2.6. Let

$$
\begin{aligned}
& \Sigma_{1}: 0 \rightarrow M_{1} \stackrel{\left[\begin{array}{l}
u_{1} \\
f_{1}
\end{array}\right]}{\longrightarrow} M_{2} \oplus N_{1} \stackrel{\left[f_{2}, u_{2}\right]}{\longrightarrow} N_{2} \rightarrow 0, \\
& \Sigma_{2}: 0 \rightarrow M_{2} \stackrel{\left[\begin{array}{l}
v_{1} \\
f_{2}
\end{array}\right]}{\longrightarrow} M_{3} \oplus N_{2} \stackrel{\left[f_{3}, v_{2}\right]}{\longrightarrow} N_{3} \rightarrow 0
\end{aligned}
$$

be short exact sequences in mod $A$. Then the sequence

$$
\Sigma_{3}: 0 \rightarrow M_{1} \stackrel{\left[\begin{array}{c}
v_{1} u_{1} \\
f_{1}
\end{array}\right]}{\longrightarrow} M_{3} \oplus N_{1} \stackrel{\left[f_{3},-v_{2} u_{2}\right]}{\longrightarrow} N_{3} \rightarrow 0
$$

is exact. Moreover, we have $\delta_{\Sigma_{3}}=\delta_{\Sigma_{1}}+\delta_{\Sigma_{2}}$. 
2.7. A short exact sequence

$$
0 \rightarrow U \stackrel{f}{\longrightarrow} W \stackrel{g}{\longrightarrow} V \rightarrow 0
$$

is said to be without isomorphism provided $f \in \operatorname{rad}(U, W)$ and $g \in \operatorname{rad}(W, V)$. Let $\Sigma: 0 \rightarrow U \stackrel{f}{\longrightarrow} W \stackrel{g}{\longrightarrow} V \rightarrow 0$ be any exact sequence. It is easy to see that if $f \in \operatorname{rad}(U, W)$, then there is an exact sequence without isomorphism $0 \rightarrow U \rightarrow$ $W^{\prime} \rightarrow V^{\prime} \rightarrow 0$ such that $W=W^{\prime} \oplus Y$ and $V=V^{\prime} \oplus Y$ for some $A$-modules $W^{\prime}, V^{\prime}$ and $Y$. Dually, if $g \in \operatorname{rad}(W, V)$, then there is an exact sequence without isomorphism $0 \rightarrow U^{\prime} \rightarrow W^{\prime} \rightarrow V \rightarrow 0$ such that $U=U^{\prime} \oplus Z$ and $W=W^{\prime} \oplus Z$ for some $A$-modules $U^{\prime}, W^{\prime}$ and $Z$. Moreover, if $\Sigma$ is nonsplittable, then there is a nonsplittable exact sequence without isomorphism $0 \rightarrow U^{\prime} \rightarrow W^{\prime} \rightarrow V^{\prime} \rightarrow 0$ such that $U=U^{\prime} \oplus Y, W=W^{\prime} \oplus Y \oplus Z$ and $V=V^{\prime} \oplus Z$ for some $A$-modules $U^{\prime}, W^{\prime}$, $V^{\prime}, Y$ and $Z$.

Lemma 2.8. Let $\Sigma: 0 \rightarrow U \stackrel{f}{\longrightarrow} W \stackrel{g}{\longrightarrow} V \rightarrow 0$ be an exact sequence without isomorphism. Then:

(i) For any nonzero direct summand $U^{\prime}$ of $U, \delta_{\Sigma}\left(U^{\prime}\right)>0$ holds.

(ii) For any nonzero direct summand $V^{\prime}$ of $V, \delta_{\Sigma}^{\prime}\left(V^{\prime}\right)>0$ holds.

Proof. (i) Let $U^{\prime}$ be a nonzero direct summand of $U$. The sequence $\Sigma$ induces an exact sequence

$$
0 \rightarrow \operatorname{Hom}_{A}\left(V, U^{\prime}\right) \rightarrow \operatorname{Hom}_{A}\left(W, U^{\prime}\right) \stackrel{f^{*}}{\longrightarrow} \operatorname{Hom}_{A}\left(U, U^{\prime}\right) .
$$

Assume that $f^{*}$ is an epimorphism. Then there is a homomorphism of $A$-modules $h: W \rightarrow U^{\prime}$ such that $f^{*}(h)=h f: U \rightarrow U^{\prime}$ is a projection. But then $f \notin$ $\operatorname{rad}(U, W)$, which yields a contradiction. Hence, $\left[V, U^{\prime}\right]-\left[W, U^{\prime}\right]+\left[U, U^{\prime}\right]>0$, and consequently $\delta_{\Sigma}\left(U^{\prime}\right)>0$.

The proof of (ii) is dual.

As a consequence of the above lemma, we get the following fact.

Lemma 2.9. Let $\Sigma: 0 \rightarrow U \rightarrow W \rightarrow V \rightarrow 0$ be a nonsplittable exact sequence. Then $\delta_{\Sigma}(U)>0$ and $\delta_{\Sigma}^{\prime}(V)>0$.

Lemma 2.10. Let $X$ be an $A$-module and $\Sigma: 0 \rightarrow U \rightarrow W \rightarrow V \rightarrow 0$ a nonsplittable short exact sequence of $A$-modules.

(i) If $\delta_{\Sigma}(X)>0$, then there exists a nonsplittable exact sequence of A-modules

$$
\Phi: 0 \rightarrow X \rightarrow Y \rightarrow V \rightarrow 0,
$$

such that $\delta_{\Phi} \leq \delta_{\Sigma}$.

(ii) If $\delta_{\Sigma}^{\prime}(X)>0$, then there exists a nonsplittable exact sequence of $A$-modules

$$
\Phi: 0 \rightarrow U \rightarrow Z \rightarrow X \rightarrow 0,
$$

such that $\delta_{\Phi} \leq \delta_{\Sigma}$.

Proof. (i) The first part of the proof is due to U. Markolf (see the proof of Theorem 4 in [7]). Let $X$ be an $A$-module such that $\delta_{\Sigma}(X)>0$. Then the last map in the following exact sequence

$$
0 \rightarrow \operatorname{Hom}_{A}(V, X) \rightarrow \operatorname{Hom}_{A}(W, X) \rightarrow \operatorname{Hom}_{A}(U, X) \rightarrow \operatorname{Ext}_{A}^{1}(V, X) \rightarrow \operatorname{Ext}_{A}^{1}(W, X)
$$

is not a monomorphism. Therefore, we find a nonsplittable exact sequence of $A$ modules $\Phi: 0 \rightarrow X \rightarrow Y \rightarrow V \rightarrow 0$, whose pullback under $W \rightarrow V$ is a splittable 
sequence. Thus we get the following commutative diagram with exact rows and columns:

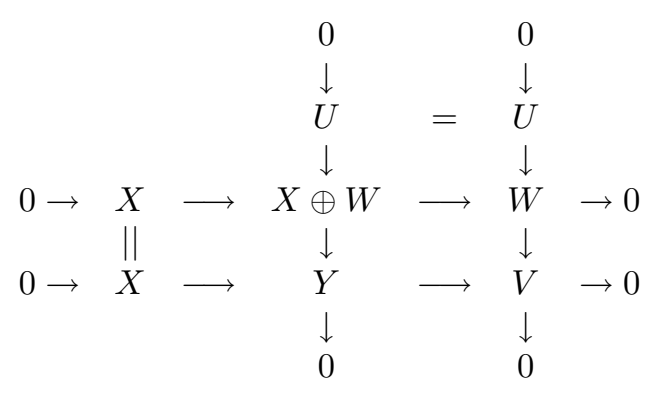

So, we have an exact sequence $\Theta: 0 \rightarrow U \rightarrow X \oplus W \rightarrow Y \rightarrow 0$. Observe that $\delta_{\Sigma}=\delta_{\Phi}+\delta_{\Theta}$. This implies that $\delta_{\Phi} \leq \delta_{\Sigma}$.

The proof of (ii) is dual.

Lemma 2.11. If $M<_{\operatorname{deg}} N$, then $\delta_{M, N}(N)>0$ and $\delta_{M, N}^{\prime}(N)>0$.

Proof. Suppose that $\delta_{M, N}^{\prime}(N)=0$. By Theorem 2.4 in [9], we know that if a module $U$ embeds into $N$ and $[U, N]=[U, M]$, then $U$ also embeds into $M$. Applying this fact for $U=N$, we obtain that $N$ embeds into $M$. But the modules $M$ and $N$ have the same dimension. This implies that $M$ is isomorphic to $N$, which gives a contradiction. Hence, $\delta_{M, N}^{\prime}(N)>0$ and $\delta_{M, N}(N)>0$ by duality.

\section{Proof of Theorems 1 and 2}

Throughout this section $A$ denotes a representation-finite algebra.

Lemma 3.1. Let $M$ and $N$ be two A-modules with $M<N$, and let

$$
\Sigma: 0 \rightarrow U \rightarrow W \rightarrow V \rightarrow 0
$$

be a short exact sequence without isomorphism in $\bmod A$ such that $\delta_{\Sigma} \leq \delta_{M, N}$. Then there exists a short exact sequence without isomorphism in $\bmod A$

$$
\Phi: 0 \rightarrow U \rightarrow Y \rightarrow Z \rightarrow 0
$$

such that $\delta_{\Sigma} \leq \delta_{\Phi} \leq \delta_{M, N}$ and $\delta_{\Phi}(Y)=\delta_{M, N}(Y)$.

Proof. Let

$$
\Sigma: 0 \rightarrow U \rightarrow W \rightarrow V \rightarrow 0
$$

be a short exact sequence of $A$-modules without isomorphism such that $\delta_{\Sigma} \leq \delta_{M, N}$. Take a short exact sequence without isomorphism in $\bmod A$,

$$
\Phi: 0 \rightarrow U \rightarrow Y \rightarrow Z \rightarrow 0
$$

such that $\delta_{\Sigma} \leq \delta_{\Phi} \leq \delta_{M, N}$, and which is maximal in the following sense. For any short exact sequence without isomorphism $\Phi^{\prime}$ in $\bmod A$ starting at $U$ and satisfying inequalities $\delta_{\Phi} \leq \delta_{\Phi^{\prime}} \leq \delta_{M, N}$, we have $\delta_{\Phi}=\delta_{\Phi^{\prime}}$. Since $\sum_{X \in \Gamma_{A}} \delta_{M, N}(X)$ is finite, such a sequence $\Phi$ exists. Assume now that $Y=Y_{1} \oplus Y_{2}$, where $Y_{1}$ is indecomposable with $\delta_{\Phi}\left(Y_{1}\right)<\delta_{M, N}\left(Y_{1}\right)$. Then $Y_{1}$ is noninjective and we have an Auslander-Reiten sequence

$$
\Sigma\left(Y_{1}\right): 0 \rightarrow Y_{1} \stackrel{h}{\longrightarrow} E \rightarrow \tau^{-} Y_{1} \rightarrow 0,
$$


and of course

$$
\Phi: 0 \rightarrow U \rightarrow Y_{1} \oplus Y_{2} \stackrel{\left(f_{1}, f_{2}\right)}{\longrightarrow} Z \rightarrow 0 .
$$

Since $f_{1} \in \operatorname{rad}\left(Y_{1}, Z\right)$, the push out of the Auslander-Reiten sequence $\Sigma\left(Y_{1}\right)$ is a splittable sequence, so we obtain the following commutative diagram with exact rows:

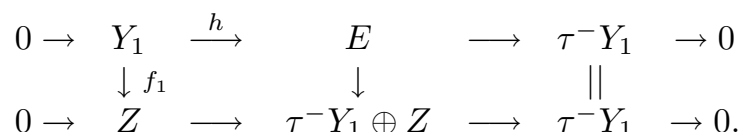

This implies that there exists a nonsplittable exact sequence

$$
\Psi: 0 \rightarrow Y_{1} \stackrel{\left(\begin{array}{c}
h \\
f_{1}
\end{array}\right)}{\longrightarrow} E \oplus Z \rightarrow \tau^{-} Y_{1} \oplus Z \rightarrow 0 .
$$

Applying Lemma 2.6 for $\Phi$ and $\Psi$, we get a new exact sequence

$$
0 \rightarrow U \stackrel{\imath}{\longrightarrow} Y_{2} \oplus E \rightarrow Z \oplus \tau^{-} Y_{1} \rightarrow 0 .
$$

Since $\Phi$ is a sequence without isomorphism, we have $\imath \in \operatorname{rad}\left(U, Y_{2} \oplus E\right)$. Hence, there is a sequence without isomorphism in $\bmod A$

$$
\Theta: 0 \rightarrow U \rightarrow \bar{Y} \rightarrow \bar{Z} \rightarrow 0,
$$

with $Y_{2} \oplus E=\bar{Y} \oplus \bar{W}$ and $Z \oplus \tau^{-} Y_{1}=\bar{Z} \oplus \bar{W}$ for some $A$-module $\bar{W}$. Thus, by Lemmas 2.6 and 2.5(i), for any $A$-module $X$ we have

$$
\delta_{\Theta}(X)=\delta_{\Phi}(X)+\delta_{\Psi}(X)=\delta_{\Phi}(X)+\delta_{\Sigma\left(Y_{1}\right)}(X)=\delta_{\Phi}(X)+\mu\left(X, Y_{1}\right) .
$$

Since $\delta_{\Phi} \leq \delta_{M, N}$ and $\delta_{\Phi}\left(Y_{1}\right) \leq \delta_{M, N}\left(Y_{1}\right)-1$, we get $\delta_{\Sigma} \leq \delta_{\Theta} \leq \delta_{M, N}$. This gives a contradiction with our choice of the sequence $\Phi$. Hence, $\delta_{\Phi}(Y)=\delta_{M, N}(Y)$, and this finishes the proof.

Lemma 3.2. If $M<N$, then $\delta_{M, N}(N)>0$ and $\delta_{M, N}^{\prime}(N)>0$.

Proof. We proceed by induction on $\sum_{X \in \Gamma_{A}} \delta_{M, N}(X)>0$. Applying equalities (2.3), we obtain $\sum_{X \in \Gamma_{A}} \delta_{M, N}(X)=\sum_{X \in \Gamma_{A}} \delta_{M, N}^{\prime}(X)$. Assume $M<N$ and that $\delta_{M, N}(N)=0$ or $\delta_{M, N}^{\prime}(N)=0$. By duality, we may assume that $\delta_{M, N}^{\prime}(N)=0$ and moreover, the modules $M$ and $N$ have no nonzero common direct summand. Let $\mathcal{F}$ be the set of all modules in $\Gamma_{A}$ which are a direct summands of $N$. Take $Y \in \mathcal{F}$. By Lemma 2.5(ii), we get

$$
\mu(N, Y)=\mu(N, Y)-\mu(M, Y) \leq \delta_{M, N}(Y)+\delta_{M, N}^{\prime}(Y)=\delta_{M, N}(Y) .
$$

So, the module $Y$ is noninjective and there is an Auslander-Reiten sequence $\Sigma(Y)$. We define a new exact sequence without isomorphism

$$
\Sigma: 0 \rightarrow N \rightarrow E(N) \rightarrow \tau^{-} N \rightarrow 0
$$

where $E(N)=\bigoplus_{Y \in \mathcal{F}} E(Y)^{\mu(N, Y)}$ and $\tau^{-} N=\bigoplus_{Y \in \mathcal{F}}\left(\tau^{-} Y\right)^{\mu(N, Y)}$. Applying Lemma 2.5(i), we obtain

$$
\delta_{\Sigma}(Y)=\mu(N, Y) \leq \delta_{M, N}(Y),
$$

for any $Y \in \Gamma_{A}$. Consequently $\delta_{\Sigma} \leq \delta_{M, N}$ and, from Lemma 3.1, there is an exact sequence without isomorphism

$$
\Phi: 0 \rightarrow N \rightarrow W \rightarrow V \rightarrow 0
$$


with $\delta_{\Phi} \leq \delta_{M, N}$ and $\delta_{\Phi}(W)=\delta_{M, N}(W)$. Then $M \oplus V \leq W$ and $\delta_{M \oplus V, W}(W)=0$. Observe that $\delta_{M, N}-\delta_{M \oplus V, W}=\delta_{\Phi}$ and, from Lemma 2.9, $\delta_{\Phi}(N)>0$. This leads to

$$
\sum_{X \in \Gamma_{A}} \delta_{M \oplus V, W}(X)<\sum_{X \in \Gamma_{A}} \delta_{M, N}(X)
$$

It follows from our inductive assumption that the modules $M \oplus V$ and $W$ are isomorphic. Then the sequence $\Phi$ has the form

$$
0 \rightarrow N \rightarrow V \oplus M \rightarrow V \rightarrow 0,
$$

and this implies that $M<_{\operatorname{deg}} N$, by Proposition 3.4 in [13]. Applying Lemma 2.11, we get $\delta_{M, N}^{\prime}(N)>0$, and hence a contradiction. This finishes the proof.

3.3. Proof of Theorem 1. Let $M$ and $N$ be $A$-modules with $M \leq N$. We may assume that $M<N$. Let $r(X)=\min \left\{\delta_{M, N}(X), \mu(N, X)\right\}$, for any $X \in \Gamma_{A}$, and let $\mathcal{F}$ be the set of all vertices of $\Gamma_{A}$ with $r(X)>0$. The set $\mathcal{F}$ does not contain injective $A$-modules and is nonempty, by Lemma 3.2. Let $N^{\prime}=\bigoplus_{X \in \mathcal{F}} X^{r(X)}=$ $\bigoplus_{X \in \Gamma_{A}} X^{r(X)}$ and $N^{\prime \prime}=\bigoplus_{X \in \Gamma_{A}} X^{\mu(N, X)-r(X)}$. Then $N=N^{\prime} \oplus N^{\prime \prime}$. We define a new exact sequence without isomorphism

$$
\Sigma: 0 \rightarrow \bigoplus_{X \in \mathcal{F}} X^{r(X)} \rightarrow \bigoplus_{X \in \mathcal{F}} E(X)^{r(X)} \rightarrow \bigoplus_{X \in \mathcal{F}}\left(\tau^{-} X\right)^{r(X)} \rightarrow 0 .
$$

Applying Lemma 2.5(i), we obtain $\delta_{\Sigma}(X)=r(X) \leq \delta_{M, N}(X)$, for any $X \in \Gamma_{A}$. Consequently, $\delta_{\Sigma} \leq \delta_{M, N}$ and, by Lemma 3.1, there is an exact sequence without isomorphism

$$
\Phi: 0 \rightarrow N^{\prime} \rightarrow W \rightarrow Z \rightarrow 0
$$

with $\delta_{\Sigma} \leq \delta_{\Phi} \leq \delta_{M, N}$ and $\delta_{\Phi}(W)=\delta_{M, N}(W)$. Then $M \oplus Z \leq N^{\prime \prime} \oplus W$ and $\delta_{M \oplus Z, N^{\prime \prime} \oplus W}(W)=0$. Let $N_{1}$ be any indecomposable direct summand of $N^{\prime \prime}$. Then $r\left(N_{1}\right)<\mu\left(N, N_{1}\right)$, and this leads to $\delta_{\Sigma}\left(N_{1}\right)=r\left(N_{1}\right)=\delta_{M, N}\left(N_{1}\right)$. Hence,

$$
\delta_{M \oplus Z, N^{\prime \prime} \oplus W}\left(N_{1}\right)=\delta_{M, N}\left(N_{1}\right)-\delta_{\Phi}\left(N_{1}\right)=\delta_{\Sigma}\left(N_{1}\right)-\delta_{\Phi}\left(N_{1}\right) \leq 0 .
$$

So, $\delta_{M \oplus Z, N^{\prime \prime} \oplus W}\left(N_{1}\right)=0$. This implies that $\delta_{M \oplus Z, N^{\prime \prime} \oplus W}\left(N^{\prime \prime}\right)=0$, and furthermore $\delta_{M \oplus Z, N^{\prime \prime} \oplus W}\left(N^{\prime \prime} \oplus W\right)=0$. Hence, $M \oplus Z \simeq N^{\prime \prime} \oplus W$, by Lemma 3.2. Finally, the sequence $\Phi$ induces an exact sequence $0 \rightarrow N^{\prime} \oplus N^{\prime \prime} \rightarrow N^{\prime \prime} \oplus W \rightarrow Z \rightarrow 0$, which has the form $0 \rightarrow N \rightarrow M \oplus Z \rightarrow Z \rightarrow 0$. In a similar way we obtain an exact sequence $0 \rightarrow Z^{\prime} \rightarrow M \oplus Z^{\prime} \rightarrow N \rightarrow 0$.

Lemma 3.4. Let $M, N$ and $X$ be A-modules such that $M<N$ and $X \in \Gamma_{A}$. Then we have:

(i) If $\delta_{M, N}^{\prime}(X)>0$, then there exist an indecomposable direct summand $N_{1}$ of $N$ and a nonsplittable exact sequence $\Phi: 0 \rightarrow N_{1} \rightarrow Y \rightarrow X \rightarrow 0$ without isomorphism such that $\delta_{\Phi} \leq \delta_{M, N}$.

(ii) If $\delta_{M, N}(X)>0$, then there exist an indecomposable direct summand $N_{1}$ of $N$ and a nonsplittable exact sequence $\Phi: 0 \rightarrow X \rightarrow Y \rightarrow N_{1} \rightarrow 0$ without isomorphism such that $\delta_{\Phi} \leq \delta_{M, N}$.

Proof. (i) Assume that $\delta_{M, N}^{\prime}(X)>0$. Applying Theorem 1 we get the exact sequence $\Sigma: 0 \rightarrow N \rightarrow M \oplus Z \rightarrow Z \rightarrow 0$, in $\bmod A$. Further, applying Lemma 2.10(ii), we obtain a nonsplittable exact sequence $\Psi: 0 \rightarrow N \rightarrow W \rightarrow X \rightarrow 0$ with $\delta_{\Psi} \leq \delta_{\Sigma}=\delta_{M, N}$. Then, by Lemma 2.9 , there is an indecomposable direct summand 
$N_{1}$ of $N$ with $\delta_{\Psi}\left(N_{1}\right)>0$. Finally, by Lemma 2.10(i), we obtain a nonsplittable exact sequence $\Phi: 0 \rightarrow N_{1} \rightarrow Y \rightarrow X \rightarrow 0$ with $\delta_{\Phi} \leq \delta_{\Psi} \leq \delta_{M, N}$.

We obtain (ii) by duality.

3.5. Proof of Theorem 2. Let $B$ be an algebra and assume that $\operatorname{Ext}_{B}^{1}(X, X)=0$ for any indecomposable $B$-module X. It is well-known that then $B$ is representationfinite. Let $M$ and $N$ be two $B$-modules with $M \leq N$. We shall show that $M \leq_{\text {ext }}$ $N$. We proceed by induction on $[N, N]-[M, M] \geq 0$. If $[N, N]-[M, M]=0$, then by Lemma 1.2 in [9], $M$ is isomorphic to $N$. Hence, we may assume that $M<N$, and that $M$ and $N$ have no common nonzero direct summand. Take any indecomposable direct summand $N_{1}$ of $N$. Applying Lemma 2.5(ii), we obtain that $\delta_{M, N}\left(N_{1}\right)+\delta_{M, N}^{\prime}\left(N_{1}\right)>0$. Without loss of generality, we may assume that $\delta_{M, N}\left(N_{1}\right)>0$. Now applying Lemma 3.4 , we get a nonsplittable exact sequence

$$
\Sigma: 0 \rightarrow N_{1} \rightarrow Y \rightarrow N_{2} \rightarrow 0
$$

with $\delta_{\Sigma} \leq \delta_{M, N}$, for some $A$-module $Y$ and some indecomposable direct summand $N_{2}$ of $N$. Since $\operatorname{Ext}_{B}^{1}\left(N_{1}, N_{1}\right)=0$, the modules $N_{1}$ and $N_{2}$ are not isomorphic. Thus, $N=N_{1} \oplus N_{2} \oplus N_{3}$, for some $A$-module $N_{3}$. Moreover, $M \leq Y \oplus N_{3}<_{\text {ext }} N$. This implies that $\left[Y \oplus N_{3}, Y \oplus N_{3}\right]<[N, N]$, by Lemma 1.2 in [9]. Then

$$
\left[Y \oplus N_{3}, Y \oplus N_{3}\right]-[M, M]<[N, N]-[M, M]
$$

and $M \leq_{\text {ext }} Y \oplus N_{3}$, by our inductive assumption. Finally, we obtain $M<_{\operatorname{ext}} N$, and this finishes the proof.

\section{REFERENCES}

[1] S. Abeasis and A. del Fra, Degenerations for the representations of a quiver of type $\mathbb{A}_{m}, \mathrm{~J}$. Algebra 93 (1985), 376-412. MR 86j: 16028

[2] I. Assem and A. Skowroński, Minimal representation-infinite coil algebras, Manuscripta Math. 67 (1990), 305-331.

[3] M. Auslander, Representation theory of finite dimensional algebras, Contemp. Math. 13 (AMS 1982), 27-39. MR 84b:16031

[4] M. Auslander and I. Reiten, Modules determined by their composition factors, Illinois J. Math. 29 (1985), 280-301. MR 86i: 16032

[5] M. Auslander, I. Reiten and S. O. Smalø, Representation Theory of Artin Algebras, Cambridge University Press (1995). MR 96c:16015

[6] K. Bongartz, A generalization of a theorem of M. Auslander, Bull. London Math. Soc. 21 (1989), 255-256. MR 90b:16031

[7] K. Bongartz, Minimal singularities for representations of Dynkin quivers, Commentari Math. Helvetici 69 (1994), 575-611. MR 96f:16016

[8] K. Bongartz, Degenerations for representations of tame quivers, Ann. Sci. École Normale Sup. 28 (1995), 647-668. MR 96i:16020

[9] K. Bongartz, On degenerations and extensions of finite dimensional modules, Advances Math. 121 (1996), 245-287. CMP 96:16

[10] K. Bongartz and P. Gabriel, Covering spaces in representation theory, Invent. Math. 65 (1982), 331-378. MR 84i:16030

[11] H. Kraft, Geometric methods in representation theory, in: Representations of Algebras, Springer Lecture Notes in Math. 944 (1982), 180-258. MR 84c:14007

[12] I. Reiten, A. Skowroński and S. O. Smalø, Short chains and short cycles of modules, Proc. Amer. Math. Soc. 117 (1993), 343-354. MR 93d:16013

[13] C. Riedtmann, Degenerations for representations of quivers with relations, Ann. Sci. École Normale Sup. 4 (1986), 275-301. MR 88b:16051

[14] C. M. Ringel, Tame Algebras and Integral Quadratic Forms, Lecture Notes in Math. 1099 (Springer 1984). MR 87f:16027 
[15] A. Skowroński and G. Zwara, On degenerations of modules with nondirecting indecomposable summands, Canad. J. Math. 48 (1996), 1091-1120. CMP 97:02

[16] G. Zwara, Degenerations in the module varieties of generalized standard Auslander-Reiten components, Colloq. Math. 72 (1997), 281-303.

[17] G. Zwara, Degenerations for modules over representation-finite biserial algebras, J. Algebra 198 (1997), 563-581. CMP 98:06

[18] G. Zwara, Degenerations for representations of extended Dynkin quivers, Comment. Math. Helvetici 73 (1998), 71-88. CMP 98:09

Faculty of Mathematics and Informatics, Nicholas Copernicus University, Chopina 12/18, 87-100 TORuń, POLAND

E-mail address: gzwara@mat.uni.torun.pl 